\title{
GROUPS WHICH SATISFY A WEAK FORM OF POINCARE DUALITY
}

\author{
by J. E. ROBERTS
}

(Received 29th March 1990)

\begin{abstract}
Our main result is that a "restricted Poincare duality" property with respect to finite dimensional coefficient modules over a field holds for a certain class of groups which includes all soluble groups of finite Hirsch length. This relies on a generalisation to the given class of a module construction by Stammbach; an extension of his result on homological dimension to these groups is given. We also generalise the well-known result that torsion-free soluble groups of finite rank are countable.
\end{abstract}

1980 Mathematics subject classification (1985 Revision): $20 \mathrm{~J} 99$.

\section{Introduction}

\subsection{Notation, definitions and statement of the main theorems}

We adopt the conventions of [2], except that we use right coefficient modules for cohomology, left for homology. $K$ will be a field, and the dimension of a $K G$-module will mean its dimension as a $K$-vector space.

We now define the property with which we are primarily concerned.

Definition. A group $G$ has restricted Poincaré duality of dimension $n$ on a subcategory ${ }_{1} \mathfrak{M}_{K G}$ of the category $\mathfrak{M}_{K G}$ of right $K G$-modules if there is a left $K G$-module $\mathscr{D}_{G}$ (called the dualizing module) such that:

(i) $\mathscr{D}_{\mathrm{G}} \cong K$ as a $K$-module, and $H_{n}\left(G, \mathscr{D}_{G}\right) \cong K$ as a $K G$-module;

(ii) $\exists[\omega] \in H_{n}\left(G, \mathscr{D}_{G}\right)$ such that the cap product

$$
H^{k}(G, M) \stackrel{\cap[\omega]}{\longrightarrow} H_{n-k}\left(G, M \otimes \mathscr{D}_{G}\right)
$$

is an isomorphism for all $k \in \mathbb{Z}$, and all objects $M$ of ${ }_{1} \mathfrak{M}_{k G}$. (We call $[\omega]$ the dualizing class).

Putting ${ }_{1} \mathfrak{M}_{K G}=\mathfrak{M}_{K G}$ here gives Poincaré duality of dimension $n$ over $K . \cap[\omega]$ is a natural transformation between the functors $H^{k}(G, M)$ and $H_{n-k}\left(G, M \otimes \mathscr{D}_{G}\right)$ from ${ }_{1} \mathfrak{O}_{K G}$ to $\mathfrak{M}_{K}$ for all $k \in \mathbb{Z}$, by naturality of cap product. (See [2, p. 146] and [3, p. 109] for elementary properties of this map.) 
Define $\int_{d} \mathfrak{P}_{K G}$ to be the full subcategory of $\mathfrak{M}_{K G}$ whose objects are the finite dimensional (f.d.) $K G$-modules (the $K$ will be omitted where it is clear which field we mean).

We now define a class of groups including all $P D^{n}$-groups, which will later be seen to have restricted Poincaré duality on ${ }_{f d} \mathfrak{M}_{K G}$.

Fix $n \in \mathbb{N}$. A group $G$ is locally orientable Poincaré duality $\left(L O P D^{n}\right)$ over $K$ if each finite subset of $G$ is contained in a subgroup of $G$ which is orientable $P D^{n}$ over $K$. If $G$ is countable $L O P D^{n}$ over $K$, there is an ascending chain of $P D^{n}$ subgroups

$$
N_{0} \leqq N_{1} \leqq \ldots \text { of } G \text { s.t. } G=\bigcup_{i \in N} N_{i}
$$

The $\left|N_{i}: N_{i-1}\right|$ are all finite ([2, Proposition 9.22]). $G$ is $P D^{n}$ if and only if $G=N_{k}$ for some $k$; otherwise, $G$ is infinitely generated.

For $G$ countable $L O P D^{n}$ over $K$, let $X_{G}$ be the set of primes $p$ s.t. given any $P D^{n}$-subgroup $S$ of $G$ there is a pair of $P D^{n}$-subgroups $P_{1}, P_{2}$ of $G$ satisfying $S \leqq P_{1}<P_{2}, p|| P_{1}: P_{2} \mid$. For any choice of ascending chain (1), $X_{G}$ is easily seen to be equal to the set of primes $p$ such that $p|| N_{i+1}: N_{i} \mid$ for infinitely many $i \in \mathbb{N}$.

Definition. Take $\mathscr{X}_{r}\left(\operatorname{resp} . \overline{\mathscr{X}}_{r}\right)$ to be the class of groups $G$ with a series

$$
G=G_{0} \geqq G_{1} \geqq \cdots \geqq G_{r}=1
$$

with each $G_{i}$ normal in $G$, and each $G_{i} / G_{i+1}$ is $L O P D^{n(i)}$ (resp. countable $L O P D^{n(i)}$ ) over $K$ with char $K$ not contained in the union of the $\mathscr{X}_{G_{t} / G_{i+1}}, 0 \leqq i \leqq r-1$. Let $\overline{\mathscr{X}}:=\bigcup_{r \in \mathrm{N}} \overline{\mathscr{X}}_{r}$, $\mathscr{X}:=\bigcup_{r \in \mathrm{N}} \mathscr{X}_{r}$. All soluble groups of finite Hirsch length lie in some $\mathscr{X}_{r}$.

Henceforth we fix a field $K$ and use the notation of (2); $X_{G}, \mathscr{X}_{r}, \bar{X}_{r}$ will always be defined with respect to this field.

Now we state the main results.

Theorem 1. If $G \in \mathscr{X}$, then $G$ has restricted Poincare duality of dimension $\sum_{i=0}^{r-1} n(i)$ on ${ }_{s d} \mathfrak{M}_{K G}$.

We will first prove this where $G$ is countable; that is, $G \in \overline{\mathscr{X}}$. We then look at groups in $\mathscr{X}$, obtaining the following, which enables us to prove Theorem 1 in general.

Theorem 2. Each $G \in X$, has a locally finite characteristic subgroup $N$ such that $G / N \in \overline{\mathscr{X}}_{r}$.

Finally, we define a property used in the proof of Theorem 1 . We will say that a group $G$ is finite dimension preserving if, for $M$ a finite dimensional $K G$-module and $k \in \mathbb{N}, H_{k}(G, M)$ and $H^{k}(G, M)$ are finite dimensional.

\subsection{Layout of paper}

In Section 1 we give some general properties of groups which have restricted Poincaré 
duality on ${ }_{f d} \mathbb{P}_{G}$ and some other subcategories of $\mathfrak{M}_{G}$. In Section 2 , the countable case of Theorem 1 is proved. Some properties of groups in $\mathscr{X}$ are given in Section 3, and we prove Theorem 2, enabling us to complete the proof of Theorem 1. In Section 4 certain groups in $\mathscr{X}$, including all soluble minimax groups, are shown to have restricted Poincaré duality on other subcategories of $\mathfrak{M}_{G}$ for some $K$. In Section 5 we consider the action of $G \in \mathscr{X}$ on the dualizing module.

\subsection{Acknowledgements}

I would like to thank: Dr P. H. Kropholler and Prof. K. W. Gruenberg, particularly the former, for many inspiring conversations and helpful comments; Professors B. Hartley and S. Donkin for pointing out several errors; Mrs A. Cook for word processing advice; and the SERC for financial support.

\section{Some properties of restricted Poincaré duality groups}

Here we give several properties of groups with restricted Poincaré duality on $\int_{d} \mathfrak{M}_{G}$, and some information on whether these hold over other subcategories of $\mathfrak{M}_{G}$. We also provide a cup product formulation of the duality for finite dimension preserving groups with restricted Poincaré duality on ${ }_{f d} \mathbb{P}_{G}$.

\subsection{Uniqueness of the dualizing module}

Suppose that $G$ has restricted Poincaré duality on ${ }_{1} \mathfrak{M}_{G}$, with $\mathscr{D}_{G}, \mathscr{D}_{G}^{\prime}$ both satisfying (i) and (ii) (see 0.1). Let $\mathscr{D}_{G}^{\prime o p}$ be the unique left $K G$-module such that $\mathscr{D}_{G}^{\prime o p} \otimes \mathscr{D}_{G}^{\prime} \cong K$. Consider $\mathscr{D}_{G} \otimes \mathscr{D}_{G}^{\prime o p}$ as a right $K G$-module via $(n \otimes m) g=g^{-1} n \otimes g^{-1} m$. If $K$ and $\mathscr{D}_{G} \otimes \mathscr{D}_{G}^{\prime o p}$ are objects of ${ }_{1} \mathfrak{M}_{G}$, then $\mathscr{D}_{G}=\mathscr{D}_{G}^{\prime}$.

As left $K G$-modules, $\left(\mathscr{D}_{G} \otimes \mathscr{D}_{G}^{\prime o p}\right) \otimes \mathscr{D}_{G}^{\prime} \cong \mathscr{D}_{G} \otimes\left(\mathscr{D}_{G}^{\prime o p} \otimes \mathscr{D}_{G}^{\prime}\right)$

$$
\operatorname{via}(m \otimes n) \otimes r \rightarrow m \otimes(n \otimes r) .
$$

Therefore

$$
K \cong H^{\circ}(G, K) \stackrel{a}{\cong} H_{n}\left(G, \mathscr{D}_{G}\right) \stackrel{\phi_{*}^{*}}{\cong} H_{n}\left(G,\left(\mathscr{D}_{G} \otimes \mathscr{D}_{G}^{\prime o p}\right) \otimes \mathscr{D}_{G}^{\prime}\right) \stackrel{\beta}{\cong} H^{o}\left(G, \mathscr{D}_{G} \otimes \mathscr{D}_{G}^{\prime o p}\right)
$$

where $\alpha, \beta$ are Poincaré duality isomorphisms. Hence $\mathscr{D}_{G} \otimes \mathscr{D}_{G}^{\prime o p} \cong K$, so $\mathscr{D}_{G} \cong \mathscr{D}_{G}^{\prime}$ as required.

The hypothesis clearly holds for ${ }_{f d} \mathfrak{M}_{G}$, hence $\mathscr{D}_{G}$ is unique for groups with restricted Poincaré duality on ${ }_{\int d} \mathbb{M}_{G}$. 


\subsection{Quotients by locally finite normal subgroups}

Let $G$ be a group with restricted Poincaré duality on ${ }_{1} \mathfrak{M}_{G}$, and $T$ a locally finite normal subgroup of $G$. Take ${ }^{T} \mathfrak{M}_{G}\left({ }_{1}^{T} \mathfrak{M}_{G}\right)$ to be the full subcategory of $\mathfrak{M}_{G}$ (respectively ${ }_{1} \mathfrak{M}_{G}$ ) with those objects on which $T$ acts trivially, and let $F$ be the natural functor ${ }^{T} \mathfrak{M}_{G} \rightarrow \mathfrak{M}_{G / T}$. Now define ${ }_{i} \mathfrak{M}_{G / T}$ to be $F\left({ }_{1}^{T} \mathfrak{M}_{G}\right)$. Then $G / T$ has restricted Poincaré duality on ${ }_{1} \mathfrak{M}_{G / T}$, as we will now show.

$K \cong H_{n}\left(G, \mathscr{D}_{G}\right)^{* *} \cong H^{n}\left(G, \mathscr{D}_{G}^{*}\right)^{*}$ (by a basic adjunction; see 1.6 for details). Hence $H^{n}\left(G, \mathscr{D}_{G}^{*}\right) \cong K$. By a spectral sequence corner argument, it follows that $H^{n}\left(G / T, H^{0}\left(T, \mathscr{D}_{G}^{*}\right)\right) \cong K$. Therefore $T$ acts trivially on $\mathscr{D}_{G}^{*}$, and hence on $\mathscr{D}_{G}$.

Let $M$ be an $K G$-module on which $T$ acts trivially. The maps

$$
\begin{aligned}
& H^{k}(G / T, M) \stackrel{\phi^{*}}{\longrightarrow} H^{k}(G, M) \\
& H_{k}(G, M) \stackrel{\phi_{*}}{\longrightarrow} H_{k}(G / T, M)
\end{aligned}
$$

induced from the natural map $\phi: G \rightarrow G / T$ are isomorphisms (by the LHS spectral sequence).

By naturality of $\cap$ with respect to change of group, the following diagram commutes.

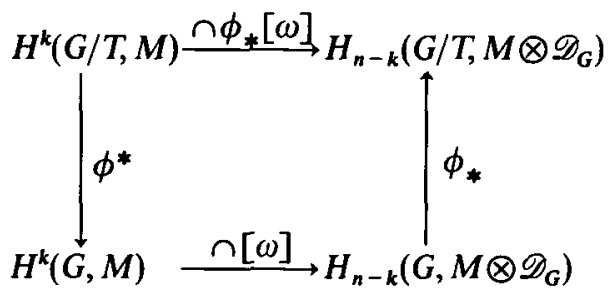

$n[\omega]$ is an isomorphism by hypothesis, hence $\cap \phi_{*}[\omega]$ also is.

Observe that where ${ }_{1} \mathfrak{M}_{G}={ }_{f d} \mathfrak{M}_{G}$, we have ${ }_{1} \mathfrak{M}_{G / T}={ }_{f d} \mathfrak{M}_{G / T}$. Hence $G / T$ has restricted Poincaré duality on finite dimensional modules if $G$ does.

\subsection{Inverse restricted Poincaré duality}

Definition. $G$ has inverse restricted Poincaré duality of dimension $n$ on ${ }_{1} \mathfrak{M}_{G}^{1}$, a subcategory of the category $\mathfrak{M}_{G}^{1}$ of left $K G$-modules, if there is a left $K G$-module $\mathscr{D}_{G}$ satisfying: 
(i) $\mathscr{D}_{G} \cong K$ as a $K$-module, and $H_{n}\left(G, \mathscr{D}_{G}\right) \cong K$ as a $K G$-module;

(ii) $\exists[\omega] \in H_{n}\left(G, \mathscr{D}_{G}\right)$ such that the modified cap product (see $[2, \mathrm{p} .147]$ for definition and properties)

$$
H^{k}\left(G, \operatorname{Hom}_{K}\left(\mathscr{D}_{G}, M\right)\right) \stackrel{\psi[\omega]}{\longrightarrow} H_{n-k}(G, M)
$$

is an isomorphism for all $k \in \mathbb{Z}$ and all objects $M$ of $\mathfrak{M}_{G}^{1}$.

Putting ${ }_{1} \mathfrak{M}_{G}^{1}=\mathfrak{M}_{G}^{1}$ here gives inverse Poincaré duality, which is equivalent to Poincare duality (in the sense that a group with one has the other, with the same dualizing module), since the dualizing module is $K$-projective (see [2, Theorem 9.4]). As in restricted Poincaré duality, $\psi[\omega]$ is a natural transformation between the functors $H^{k}\left(G, \operatorname{Hom}_{K}\left(\mathscr{D}_{G},{ }_{-}\right)\right)$and $H_{n-k}(G, \ldots)$ from ${ }_{1} \mathfrak{P}_{G}^{1}$ to $\mathfrak{M}^{1}$.

$\psi$ is defined by

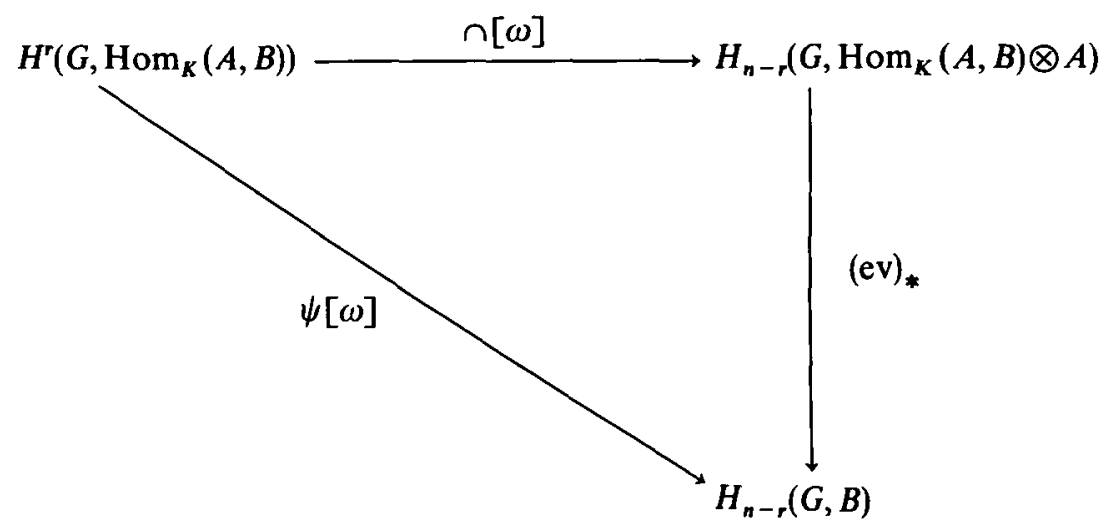

where ev: $\operatorname{Hom}_{K}(A, B) \otimes A \rightarrow B$ is given by

$$
f \otimes a \rightarrow f(a)
$$

and $[\omega] \in H_{n}(G, A)$.

Where $A=\mathscr{D}_{G}$, it is easy to see that ev is an isomorphism.

Lemma 1. (a) Suppose $G$ has restricted Poincaré duality on ${ }_{1} \mathfrak{M}_{G}$, with dualizing module $\mathscr{D}_{G}$. Then $G$ has inverse restricted duality with dualizing module $\mathscr{D}_{G}$ on any subcategory ${ }_{2} \mathfrak{M}_{G}^{1}$ of $\mathfrak{M}_{G}^{1}$ in which each object $M$ satisfies $\operatorname{Hom}_{K}\left(\mathscr{D}_{G}, M\right)$ an object of $\mathfrak{M}_{G}$.

b) Suppose $G$ has inverse restricted Poincaré duality on ${ }_{1} \mathfrak{M}_{G}^{1}$, with dualizing module $\mathscr{D}_{G}$. Then $G$ has restricted Poincaré duality with dualizing module $\mathscr{D}_{G}$ on any subcategory ${ }_{2} \mathbb{P}_{G}$ of $\mathfrak{M}_{G}$ in which each object $M$ satisfies $\operatorname{Hom}_{K}\left(\mathscr{D}_{G}^{o p}, M\right)$ an object of $\mathfrak{M}_{G}^{1}$. 
Proof. (a) Trivial.

(b) Substitute $\operatorname{Hom}_{K}\left(\mathscr{D}_{G}^{o p}, M\right)$ for $B$ in (3). There is a natural isomorphism $\operatorname{Hom}_{K}\left(\mathscr{D}_{G}, \operatorname{Hom}_{K}\left(\mathscr{D}_{G}^{o p}, M\right)\right) \rightarrow \operatorname{Hom}_{K}\left(\mathscr{D}_{G} \otimes \mathscr{D}_{G}^{o p}, M\right) \cong M$ given by a basic adjunction. Applying this, we obtain the result.

Where $M$ is a f.d. left $K G$-module, $\operatorname{Hom}_{K}\left(\mathscr{D}_{G}, M\right)$ is a f.d. right $K G$-module, and where $M$ is a f.d. right $K G$-module, $\operatorname{Hom}_{K}\left(\mathscr{D}_{G}^{o p}, M\right)$ is a f.d. left $K G$-module. Hence $G$ has inverse restricted Poincaré duality on f.d. modules if and only if $G$ has restricted Poincaré duality on f.d. modules, and, if both hold, the dualizing modules are the same.

\subsection{Duality groups with restricted Poincaré duality}

All soluble groups s.t. $\operatorname{cd}_{\mathbf{Z}} G=\operatorname{hd}_{\mathbf{Z}} G<\infty$ are duality groups over $\mathbb{Z}$ and hence over $\mathbb{Q}$ by [8]. We will see that these groups are also restricted Poincaré duality on finite dimensional modules. Many of them, $\mathbb{Z}[1 / 2] 1\langle x 2\rangle$ for instance, are not Poincaré duality groups. However, there is a connection between the duality and the restricted Poincaré duality on a subcategory of $\mathfrak{M}_{G}$ for some groups with both properties.

Lemma 2. Let $G$ be a duality group with dualizing module $\mathscr{D}_{\mathrm{G}}$. The following are equivalent:

(i) $G$ has restricted Poincaré duality of dimension $n$ on ${ }_{1} \mathfrak{M}_{G}$ with dualizing module $\mathscr{D}_{G}$ such that $\mathscr{D}_{G}^{o p}$ is an object of ${ }_{1} \mathfrak{M}_{G}$.

(ii) There is a $K G$-module $\mathscr{D}_{G} \stackrel{K}{\cong} K$ s.t. $\mathscr{D}_{G}^{o p}$ is an object of ${ }_{1} \mathfrak{M}_{G}$, and a $K G$-module homomorphism $\quad \phi: D_{G} \rightarrow \mathscr{D}_{G} \quad$ which induces isomorphisms $\phi_{*}: H_{r}\left(G, M \otimes D_{G}\right) \rightarrow$ $H_{r}\left(G, M \otimes \mathscr{D}_{G}\right)$ for all $r \in \mathbb{N}$ and all objects $M$ of ${ }_{1} \mathfrak{M}_{G}$.

Proof. (ii) $\Rightarrow$ (i): Follows easily from naturality of cap product with respect to coefficient homomorphisms.

(i) $\Rightarrow$ (ii): Define $\rho: G \rightarrow K$ by $\rho(g)=g .1$ in $\mathscr{D}_{G}$. Let $J$ be the two-sided ideal of $K G$ generated by the $\rho(g)-g, g \in G$. Then

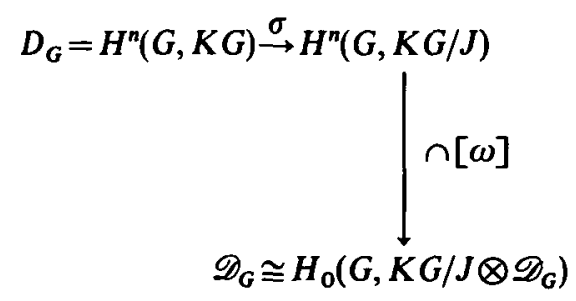

where $[\omega]$ is the dualizing class of $H_{n}\left(G, \mathscr{D}_{\mathrm{G}}\right)$ (see 0.1 ).

$\sigma$ is onto since $c d_{K} G=n$. $K G / J$ regarded as a right module is $\mathscr{D}_{G}^{\circ p}$, so the vertical map 
is an isomorphism. So we have $\phi: D_{G} \rightarrow \mathscr{D}_{G}$. By naturality of $\cap$, the following diagram commutes.

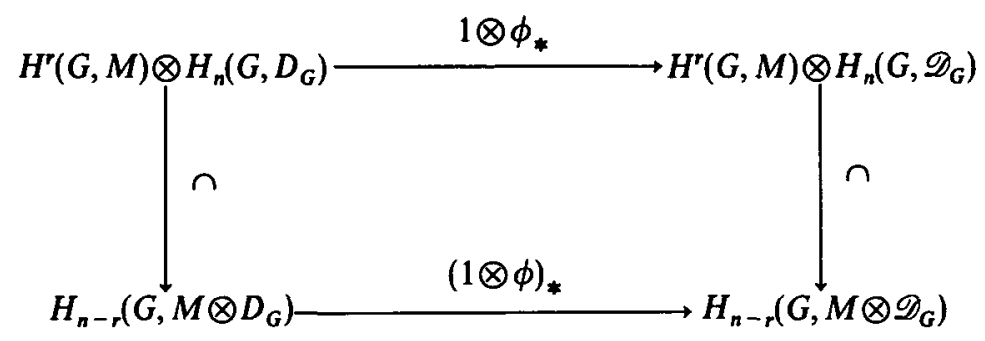

Where $M$ is an object of ${ }_{1} \mathfrak{M}_{G}$, the $\cap$ maps are both isomorphisms, so it suffices to prove that $\phi_{*}: H_{n}\left(G, D_{G}\right) \rightarrow H_{n}\left(G, \mathscr{D}_{G}\right)$ is an isomorphism. By [2, Lemma 9.1] the following diagram commutes and the vertical maps are isomorphisms.

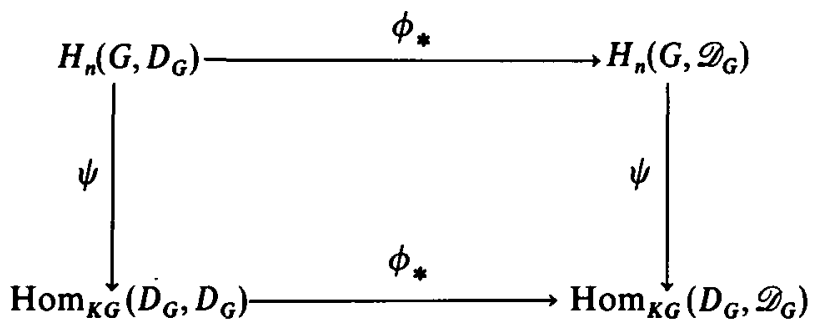

The bottom $\phi_{*}$ is nonzero, hence the top $\phi_{*}$ is an isomorphism.

Note that an analogous result holds for restricted inverse Poincaré duality, where $G$ is an inverse duality group.

Observe that not all duality groups have restricted Poincaré duality on ${ }_{f d} \mathbb{M}_{G}$; the free group on two generators is a duality group, but does not have restricted Poincare duality on this category.

\subsection{The cap product formulation}

Corollary. Suppose $G$ has restricted Poincaré duality on ${ }_{f d} \mathfrak{M}_{G}$ and $G$ is finite dimension preserving. Then for all finite dimensional $K G$-modules $M$, the map

$$
H^{r}(G, M) \otimes H^{n-r}\left(G, \operatorname{Hom}_{K}\left(M, \mathscr{D}_{G}\right)\right) \stackrel{(\mathrm{ev}) * \circ \cup}{\longrightarrow} H^{n}\left(G, \mathscr{D}_{G}\right)
$$

is an exact pairing, where ev is the map 


$$
M \otimes \operatorname{Hom}_{K}\left(M, \mathscr{D}_{G}^{*}\right) \rightarrow \mathscr{D}_{G}^{*}
$$

given by $m \otimes \phi \rightarrow m \phi$.

Proof. Take $L$ any group, $V$ any $K L$-module. Let

$$
\cdots \rightarrow P_{i} \rightarrow P_{i-1} \rightarrow \cdots \rightarrow P_{0} \rightarrow K
$$

be a projective resolution for $K$ over $K L$.

Applying $\operatorname{Hom}_{K L}\left(, \operatorname{Hom}_{K}(V, K)\right)$ to this gives a cochain complex whose cohomology is $H^{*}\left(L, V^{*}\right)$. Using $\operatorname{Hom}_{K}\left(\bigotimes_{K L} V, K\right)$ instead gives a cochain complex with cohomology $H_{*}(L, V)^{*}$. The basic adjunction

$$
\operatorname{Hom}_{K L}\left(, \operatorname{Hom}_{K}(V, K)\right) \rightarrow \operatorname{Hom}_{K}\left(\otimes_{K L} V, K\right)
$$

gives an isomorphism between the cochain complexes which induces the isomorphism

$$
H^{*}\left(L, V^{*}\right) \stackrel{\alpha}{\rightarrow} H_{*}(L, V)^{*}
$$

Now suppose $G$ satisfies restricted Poincaré duality on ${ }_{f d} \mathfrak{M}_{G}$ and is finite dimension preserving. Let $M$ be a finite dimensional module. Consider the diagram

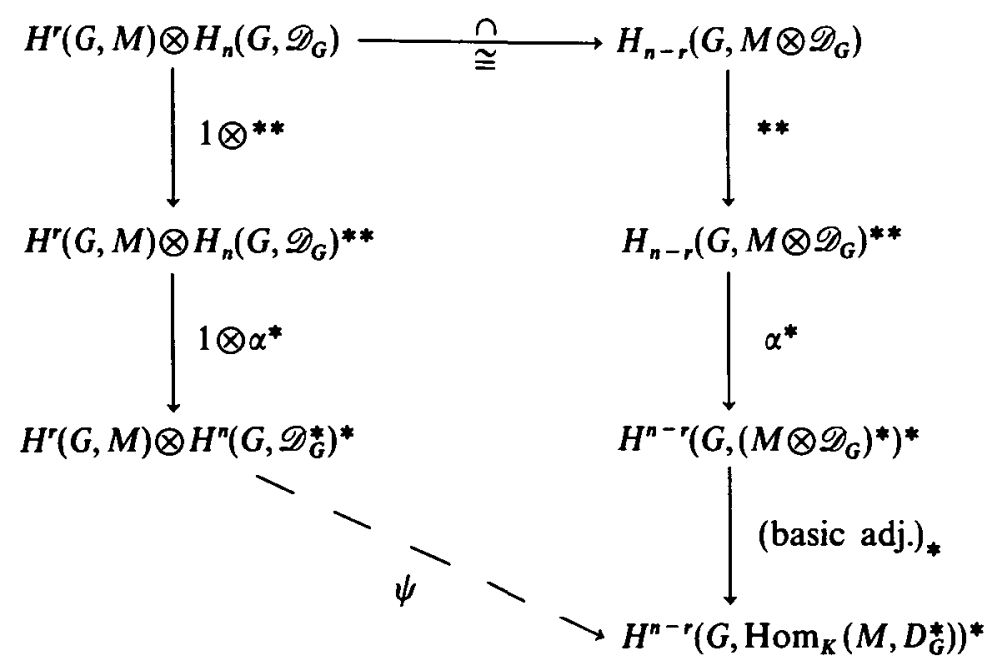

As all the other maps are isomorphisms, $\phi$ may be defined by this diagram; it too is clearly an isomorphism. Let $\theta$ be defined by 


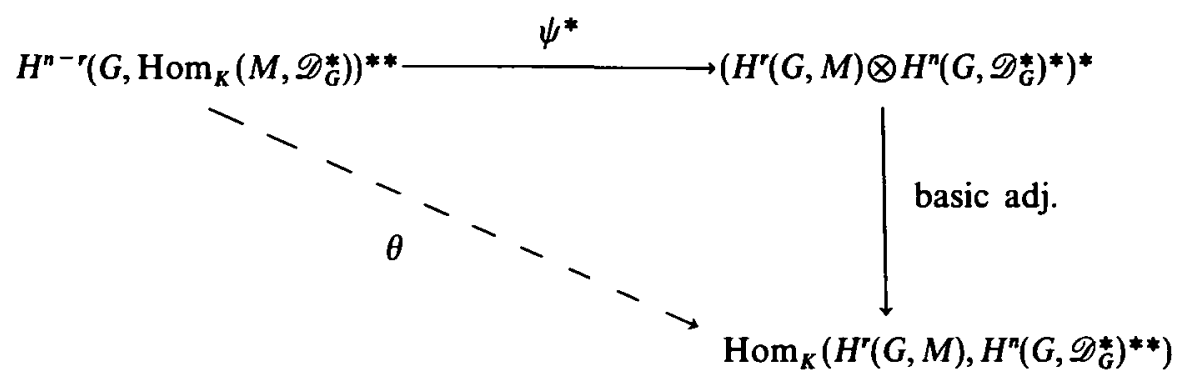

Define $\beta: H^{r}(G, M) \otimes H^{n-r}\left(G, \operatorname{Hom}_{K}\left(M, \mathscr{D}_{G}^{*}\right)\right)^{* *} \rightarrow H^{n}\left(G, \mathscr{D}_{G}^{*}\right)^{* *}$ by

$$
\beta(v \otimes w)=\dot{v}(w \theta)
$$

Taking bases of the $K$-vector spaces involved, then performing a routine calculation, it can be seen that the following diagram commutes.

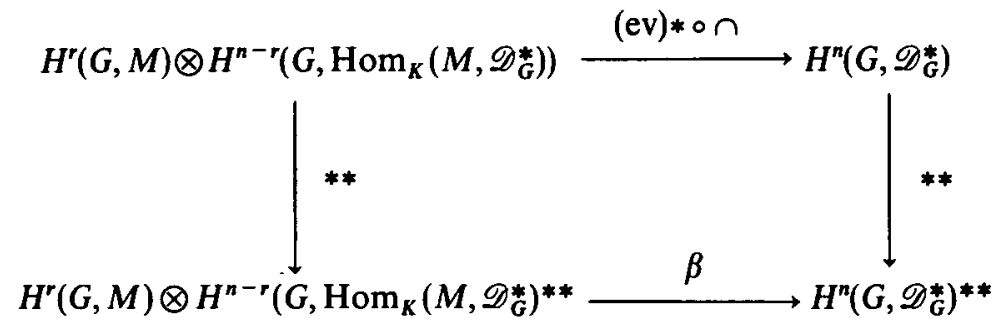

The result follows.

\section{Proof of the countable case of Theorem 1}

We prove by induction on $r$ that if $G \in \bar{X}$, then $G$ is finite dimension preserving and has restricted Poincaré duality on finite dimensional modules.

\subsection{Proof for $r=1$}

A $P D^{n}$ group (over $K$ ) plainly has restricted Poincaré duality ồ ${ }_{f d} \mathfrak{M}_{G}$, and is finite dimension preserving because it is of type (FP). Throughout this section, $M$ will be a f.d. $K G$-module.

Suppose

$$
G=\bigcup_{i=0}^{\infty} N_{i}, \quad N_{0} \leqq N_{1} \leqq \cdots
$$

with the $N_{i}$ all orientable $P D^{n}$. There is a short exact sequence of $K G$-modules 


$$
\stackrel{\lim }{\longleftarrow}{ }^{(1)} H^{k-1}\left(N_{i}, M\right) \rightarrow H^{k}(G, M) \rightarrow \stackrel{\lim }{\longleftarrow} H^{k}\left(N_{i}, M\right)
$$

(see [4]) where $\stackrel{\lim }{\longleftarrow}, \stackrel{\lim }{\longleftarrow}$ (1) have been taken over

$$
\left(\cdots \stackrel{\operatorname{Res}}{\longrightarrow} H^{s}\left(N_{i}, M\right) \stackrel{\operatorname{Res}}{\longrightarrow} H^{s}\left(N_{i-1}, M\right) \stackrel{\operatorname{Res}}{\longrightarrow} \cdots \stackrel{\operatorname{Res}}{\longrightarrow} H^{s}\left(N_{0}, M\right)\right)
$$

for $s=k, s=k-1$ respectively.

There is also an isomorphism

$$
\stackrel{\lim }{\longrightarrow} H_{k}\left(N_{i}, M\right) \rightarrow H_{k}(G, M)
$$

where $\stackrel{\lim }{\longrightarrow}$ is taken over the system

$$
\left(H_{k}\left(N_{0}, M\right) \stackrel{\text { Cor }}{\longrightarrow} H_{k}\left(N_{1}, M\right) \stackrel{\text { Cor }}{\longrightarrow} \cdots \stackrel{\text { Cor }}{\longrightarrow} H_{k}\left(N_{i}, M\right) \stackrel{\text { Cor }}{\longrightarrow} \cdots\right)
$$

As automorphisms of $H_{n}\left(N_{i}, M\right)$ or $H^{n}\left(N_{i}, M\right)$, it is well known that

$$
\text { Cor } \circ \operatorname{Res}=x\left|N_{i}: N_{i-1}\right|
$$

char $K \notin X_{G}$, so $x\left|N_{i}: N_{i-1}\right|$ is an isomorphism, for sufficiently large $i$. $H_{n}\left(N_{i}, K\right) \stackrel{K N_{i}}{\cong} K$ for all $i \in \mathbb{N}$, since the $N_{i}$ are orientable $P D^{n}$. The Cor maps in (5) are onto by (6), hence $H_{n}(G, K) \cong K$. $K$.

The duality isomorphism in the orientable $P D^{n}$ groups is given by a cap product, which may be regarded [2] as a map

$$
H_{n}\left(N_{i}, K\right) \rightarrow \operatorname{Hom}_{K N_{i}}\left(H^{k}\left(N_{i}, M\right), H_{n-k}\left(N_{i}, M\right)\right)
$$

which is natural with respect to changes of group and module. Choose an element $[\omega]$ of $H_{n}(G, K)$ and let $\left[\omega_{i}\right]$ be its image under restriction to $H_{n}\left(N_{i}, K\right)$. The following commutes, since $n$ is natural in the group. 


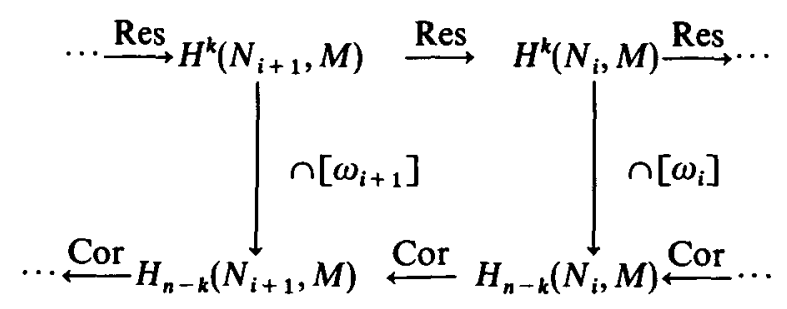

By (6), the Res maps are $(1-1)$ and the Cor maps are onto. The $N_{i}$ are finite dimension preserving, so all the modules in (7) are finite dimensional. Hence $\exists n_{0} \in \mathbb{N}$ such that

$$
\text { Res: } \begin{aligned}
H^{k} & \left(N_{l+1}, M\right) \rightarrow H^{k}\left(N_{l}, M\right) \text { and Cor: } H_{n-k}\left(N_{l}, M\right) \\
& \rightarrow H_{n-k}\left(N_{l+1}, M\right) \text { are isomorphisms for } l>n_{0} .
\end{aligned}
$$

The vertical maps therefore induce an isomorphism

$$
\psi: \stackrel{\lim }{\longleftarrow} H^{k}\left(N_{i}, M\right) \rightarrow \stackrel{\lim }{\longrightarrow} H_{n-k}\left(N_{i}, M\right)
$$

which is the unique such $K G$-module isomorphism satisfying $\left.\psi\right|_{N_{i}}=\cap\left[\omega_{i}\right]$. $\lim ^{(1)} H^{k-1}\left(N_{i}, M\right)=0$ by the Mittag-Leffler condition (see [1]), satisfied since all the $H^{k-1}\left(N_{i}, M\right)$ are finite dimensional. It follows that $\alpha$ is an isomorphism, and $\psi$ is the unique isomorphism $H^{k}(G, M) \rightarrow H_{n-k}(G, M)$ such that $\left.\psi\right|_{N_{i}}=\cap\left[\omega_{i}\right]$. But $\left.\cap[\omega]\right|_{N_{i}}=$ $\cap\left[\omega_{i}\right]$, hence $\psi=\cap[\omega]$. Therefore $\cap[\omega]$ is an isomorphism, so $G$ has restricted Poincaré duality on finite dimensional modules, with dualizing module $K$.

By (8), $\operatorname{dim}_{K}\left(\stackrel{\lim }{\longleftarrow} H^{k}\left(N_{i}, M\right)\right)=\operatorname{dim}_{K}\left(H^{k}\left(N_{l}, M\right)\right) \quad$ and $\quad \operatorname{dim}_{K}\left(\stackrel{\lim }{\longrightarrow} H_{n-k}\left(N_{i}, M\right)\right)=$ $\operatorname{dim}_{K}\left(H^{k}\left(N_{l}, M\right)\right)$. Hence $G$ is finite dimension preserving.

\subsection{The induction step}

Throughout this part, $G \in \bar{X}_{r}$, the notation of (2) is used, and $N:=G_{1}$. Assume result true for $G \in \bar{X}_{r-1}$ (hence for $N$ ).

\subsection{1 $G$ is finite dimension preserving}

For the extension $N \rightarrow G \rightarrow G / N$, there is a Lyndon-Hochschild-Serre spectral sequence

$$
H^{k-l}\left(G / N, H^{l}(N, M)\right) \Rightarrow H^{k}(G, M) .(\text { see }[5],[9] \text {.) }
$$


So $H^{k}(G, M)=S_{k} \geqq S_{k-1} \geqq \cdots \geqq S_{0}=1$ where $S_{i} / S_{i-1}$ is a section of $H^{k-i}\left(G / N, H^{i}(N, M)\right)$.

The $H^{k-i}\left(G / N, H^{i}(N, M)\right)$ are finite dimensional, since $N$ and $G / N$ are finite dimension preserving by the induction hypothesis. Therefore $H^{k}(G, M)$ is finitely generated. A similar argument may be used on homology.

2.2.2 Construction of the dualizing module in the general case

Let $L_{i}:=H_{n(i)}\left(G_{i} / G_{i+1}, K\right)$. $G$ acts on $L_{i}$ via conjugation on $G_{i} / G_{i+1}$. Set $\mathscr{D}_{G}:=$ $L_{0}^{o p} \otimes \cdots \otimes L_{r-1}^{o p}$. From (1.1) we know that $\mathscr{D}_{G} \cong K$ as a $K$-module. By the method of [2, Lemma 7.13], it can be shown that $H_{n}\left(G, \mathscr{D}_{G}\right) \cong K$ as a $K G$-module.

\subsubsection{The induction argument}

Set $l:=n(1)+n(2)+\cdots+n(r-1)$.

\section{Lemma 3.}

$$
H^{k}(N, M) \stackrel{\cap[\omega]}{\longrightarrow} H_{l-k}\left(N, M \otimes D_{G}\right)
$$

is a $K G$-module isomorphism, where $[\omega] \in H_{l}\left(N, D_{G}\right)$.

Proof. $\operatorname{Res}_{N}^{G}\left(\mathscr{D}_{G}\right)=\mathscr{D}_{N}$, so the given map is a $K N$-module isomorphism by the induction hypothesis. It now suffices to show that it is also a $K G$-module homomorphism. Since $\cap$ is natural with respect to group homomorphisms, the diagram below commutes,

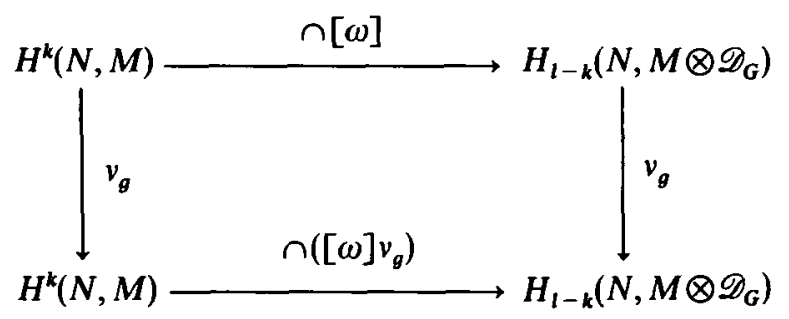

where $v_{g}$ are the natural maps in homology given by the action of $g \in G$.

$$
H_{1}\left(N, \mathscr{D}_{G}\right) \stackrel{K N}{\cong} H_{l}\left(N, \mathscr{D}_{N}\right) \cong K
$$

$K \cong H_{n}\left(G, \mathscr{D}_{G}\right) \cong H_{n-l}\left(G / N, H_{l}\left(N, \mathscr{D}_{G}\right)\right) \cong H_{l}\left(N, \mathscr{D}_{G}\right)^{G / N}$ (by result for $\left.r=1\right)$. Hence $G$ acts trivially on $H_{N}\left(N, \mathscr{D}_{G}\right)$ so $[\omega] v_{g}=[\omega]$. Therefore $\cap[\omega]$ is a $K G$-module isomorphism as required. 


$$
\text { Let } \cdots \stackrel{\partial_{1}}{\longrightarrow} P_{3} \stackrel{\partial_{1}}{\longrightarrow} P_{2} \stackrel{\partial_{1}}{\longrightarrow} P_{1} \stackrel{\partial_{1}}{\longrightarrow} P_{0} \rightarrow K
$$

be a projective resolution for $K$ over $K G$, and let

$$
\stackrel{\partial_{2}}{\longrightarrow} Q_{3} \stackrel{\partial_{2}}{\longrightarrow} Q_{2} \stackrel{\partial_{2}}{\longrightarrow} Q_{1} \stackrel{\partial_{2}}{\longrightarrow} Q_{0} \rightarrow K
$$

be a projective resolution for $K$ over $K G / N$.

Using these, we form two double cochain complexes of the form given below. In (I), $X_{i, j}=\operatorname{Hom}_{G / N}\left(Q_{i}, \operatorname{Hom}_{N}\left(P_{j}, M\right)\right)$ and the differentials are $\partial^{\prime}=\partial_{2}^{*}, \partial^{\prime \prime}=(-1)^{i} \partial_{1 *}^{*}$. In (II), $X_{i, j}=Q_{n-l-i} \otimes_{G / N} P_{l-j} \otimes_{N}\left(M \otimes \mathscr{D}_{G}\right)$ and the differentials are $\partial^{\prime}=\dot{\partial}_{2^{*}}, \partial^{\prime \prime}=(-1)^{i} \partial_{1 * *}$ It is well known that I and II give the Lyndon-Hochschild-Serre spectral sequences for cohomology and homology respectively. (See $[5,9]$ for details.)

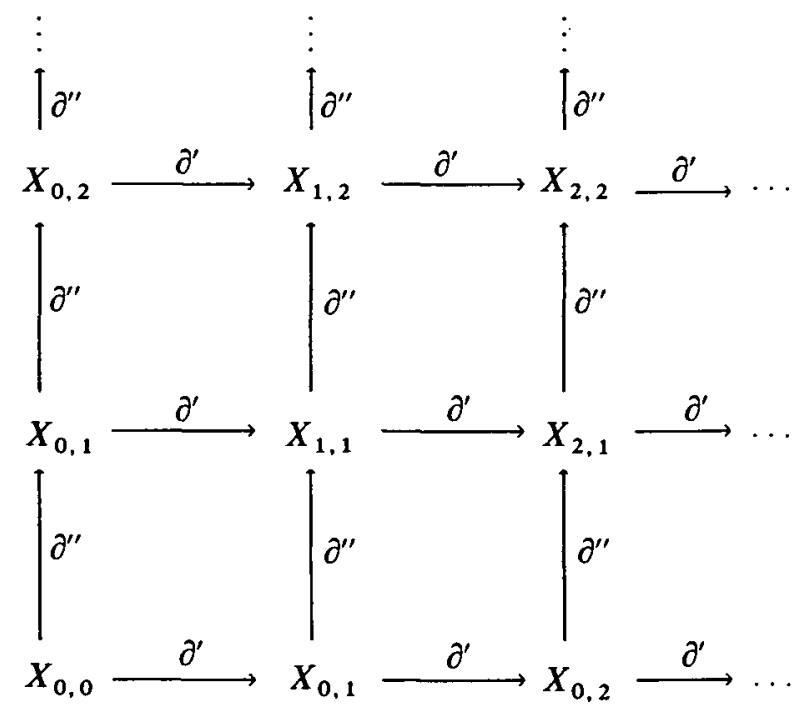

Let $\omega$ be a representative cycle of a nonzero element of $H_{n}\left(G, \mathscr{D}_{G}\right)$ and take $\alpha \omega$ to be a representative cycle of the corresponding element of $H_{n-l}\left(G / N, H_{l}\left(N, \mathscr{D}_{G}\right)\right)$ under the spectral sequence corner isomorphism.

On the chain level, $n$ is natural with respect to module homomorphisms; by this and Lemma 3 we may define a map from double complex (I) to double complex (II) by $n * \circ \cap(\alpha \omega)$.

Consider the filtration

$$
{ }_{1} F^{p}\left((\operatorname{Tot} X)^{n}\right)=\bigoplus_{\substack{r+s=n \\ r \geqq p}} X_{r, s}
$$


The spectral sequences associated with this for (I) and (II) respectively satisfy

$$
\begin{gathered}
\text { (l) } E_{2}^{p, q}=H^{p}\left(G / N, H^{q}(N, M)\right) \\
\text { (II) }_{1}^{p} E_{2}^{p, q}=H_{n-l-p}\left(G / N, H_{l-q}\left(N, M \otimes \mathscr{D}_{G}\right)\right) .
\end{gathered}
$$

The map between these induced from $n * \circ \cap(\alpha \omega)$ is $n * \circ \cap[\alpha \omega]$, which is an isomorphism where $M$ is finite dimensional, by the induction hypothesis. By the mapping theorem for spectral sequences, this induces an isomorphism between the graded objects associated with the $H^{n}(\operatorname{Tot} X)$ suitably filtered for the two spectral sequences.

Now consider the filtration

$$
\begin{aligned}
& { }_{2} F^{q}\left((\operatorname{Tot} X)^{m}\right)=\bigoplus_{\substack{r+s=m \\
s \geqq q}} X_{r, s^{*}} \\
& \text { (1) } E_{1}^{p \cdot q}=\left\{\begin{array}{l}
0 \text { for } p \neq 0 ; \\
H^{m}(G, M) \text { for } p=0 .
\end{array}\right. \\
& { }_{2}^{(11)} E_{2}^{p, q}=\left\{\begin{array}{l}
0 \text { for } p \neq n-l \\
H_{m}\left(G, M \otimes \mathscr{D}_{G}\right) \text { for } p=n-l .
\end{array}\right.
\end{aligned}
$$

The $E_{\infty}$-page is clearly the $E_{2}$-page, in both cases. Hence $\cap * 0 \cap(\alpha \omega)$ induces an isomorphism

$$
\theta: H^{p}(G, M) \rightarrow H_{n-p}\left(G, M \otimes \mathscr{D}_{G}\right)
$$

It remains only to check that $\theta=\cap[\omega]$. As both maps are natural maps from a universal cohomology theory for $G$ to a cohomology theory for $G$, we need only ensure that they coincide for $p=0$; that is, that the following diagram commutes.

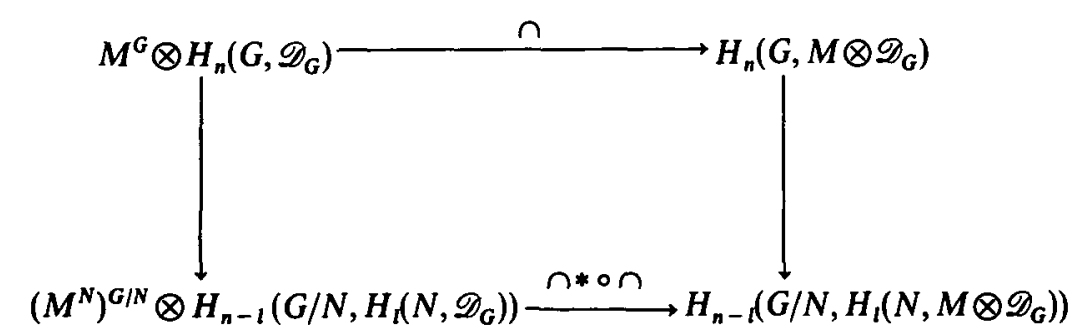

where the vertical maps are spectral sequence corner isomorphisms.

It is easily seen that 


$$
M^{G} \otimes H_{n}\left(G, \mathscr{D}_{G}\right) \rightarrow H_{n}\left(G, M \otimes \mathscr{D}_{G}\right)
$$

is given by $m \cap s=f_{m}(s),\left(s \in H_{n}\left(G, \mathscr{D}_{G}\right)\right)$ where $f_{m}: H_{n}\left(G, \mathscr{D}_{G}\right) \rightarrow H_{n}\left(G, M \otimes \mathscr{D}_{G}\right)$ is induced from the module homomorphism $v_{m}: \mathscr{D}_{G} \rightarrow M \otimes \mathscr{D}_{G}$ given by

$$
e \rightarrow m \otimes e .
$$

Similarly, $\left(M^{N}\right)^{G / N} \otimes H_{n-l}\left(G / N, H_{l}\left(N, \mathscr{D}_{G}\right)\right) \stackrel{\cap * \circ \cap}{\longrightarrow} H_{n-l}\left(G / N, H_{l}\left(N, M \otimes \mathscr{D}_{G}\right)\right)$ is given by $m(\cap * \circ \cap) s=f_{m}(s)$ where

$$
f_{m}: H_{n-l}\left(G / N, H_{l}\left(N, \mathscr{D}_{G}\right)\right) \rightarrow H_{n-l}\left(G / N, H_{l}\left(N, M \otimes \mathscr{D}_{G}\right)\right)
$$

is induced from $v_{m}$.

It now only remains to check that the following diagram commutes.

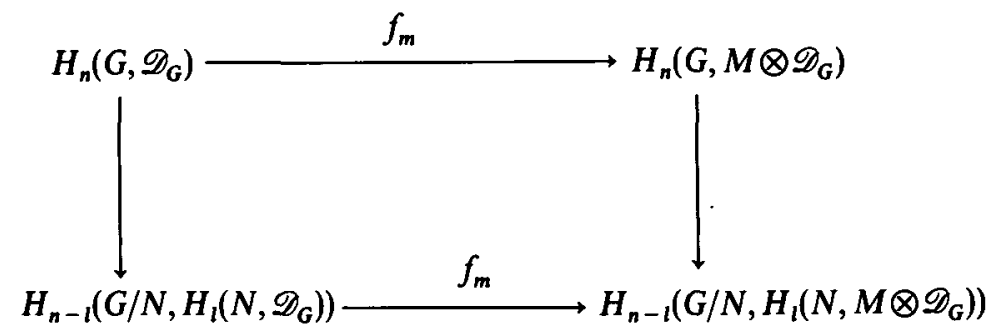

where the vertical maps are spectral sequence corner isomorphisms. But these commute with module homomorphisms, and the result follows.

\section{Some properties of groups in $\mathscr{X}$, and the end of the proof of Theorem 1}

\subsection{Proof of Theorem 2}

Definition. We will say that a group $G$ is $L P D^{n}$ if each finite subset of $G$ is contained in a $P D^{n}$-subgroup of $G$.

Some observations on the $P D^{n}$ subgroups of an $L O P D^{n}$ group $G$

Write $\mathscr{P}$ for the set of all $P D^{n}$ subgroups of $G$. Where $P \in \mathscr{P}, P$ is generated by a finite subset $X$ of $G$. If $Y$ is another finite subset of $G, X \cup Y$ lies in some $P_{1} \in \mathscr{P}$ containing $P$. It follows from the remark after (1), that

(i) Given $g \in G \exists k \in \mathbb{N}$ s.t. $g^{k} \in P$.

(ii) $N_{G}(P) / P$ is locally finite.

If $X, Y$ are finite generating sets for $P_{1}, P_{2} \in \mathscr{P},\left\langle P_{1}, P_{2}\right\rangle$ is generated by $X \cup Y$, so lies in some $P_{3} \in \mathscr{P}$. Hence $\left|\left\langle P_{1}, P_{2}\right\rangle: P_{1}\right|,\left|\left\langle P_{1}, P_{2}\right\rangle: P_{2}\right|$ are finite, giving $\left|P_{1}: P_{1} \cap P_{2}\right|$ finite. By [2, Theorem 9.9], $P_{1} \cap P_{2}$ is $P D^{n}$. Hence 
(iii) $P_{1}, P_{2}, \ldots, P_{n} \in \mathscr{P} \Rightarrow P_{1} \cap P_{2} \cap \cdots \cap P_{n} \in \mathscr{P}$.

$C_{G}(P) /\left(C_{G}(P) \cap P\right) \cong C_{G}(P) \cdot P / P \leqq N_{G}(P) / P \quad$ and $C_{G}(P) \cap P \leqq \zeta\left(C_{G}(P)\right) \quad$ hence $C_{G}(P) / \zeta\left(C_{G}(P)\right)$ is locally finite. By Schur's Theorem,

(iv) $\left(C_{G}(P)\right)^{\prime}$ is locally finite.

Lemma 4. $S:=\bigcup_{P_{E} \mathscr{P}} C_{G}(P)$ is a characteristic subgroup of $G, L:=\bigcup_{P_{\mathcal{P}}}\left(C_{G}(P)\right)^{\prime}$ is a locally finite characteristic subgroup of $G$ and $S^{\prime}=L$.

Proof of lemma. Where $P_{1}, P_{2}, \ldots, P_{n}$ are $P D^{n}$ subgroups of $G$, $\left\langle C_{G}\left(P_{1}\right), \ldots, C_{G}\left(P_{n}\right)\right\rangle \subseteq C_{G}\left(P_{1} \cap \cdots \cap P_{n}\right)$, hence $S<G$. Plainly $S$ is characteristic in $G$. $\left\langle\left(C_{G}\left(P_{1}\right)\right)^{\prime}, \ldots,\left(C_{G}\left(P_{n}\right)\right)^{\prime}\right\rangle \subseteq\left\langle\left(C_{G}\left(P_{1}\right), \ldots, C_{G}\left(P_{n}\right)\right\rangle^{\prime} \subseteq\left(C_{G}\left(P_{1} \cap \cdots \cap P_{n}\right)\right)^{\prime} \quad\right.$ hence $\quad L<G$; clearly $L$ is characteristic in $G$. Any subset of $L$ lies in some $\left(C_{G}(P)\right)^{\prime}$, hence $L$ is locally finite. From $\left\langle C_{G}\left(P_{1}\right), C_{G}\left(P_{2}\right)\right\rangle \subseteq\left(C_{G}\left(P_{1} \cap P_{2}\right)\right)^{\prime}$, it follows that $S^{\prime}=L$.

Proof of Theorem 2 for $r=1$. $S / L$ is abelian. The torsion elements of $S / L$ therefore generate a locally finite subgroup $W / L . W$ is a locally finite subgroup of $S$, and $S / W$ is torsion free abelian.

We will now show that $G / W$ is countable. $G / W$ will then be in $\bar{X}_{1}$ by 1.2 .

Choose a $P D^{n}$ subgroup $N$ of $G$. Given $g \in G$, let $N_{g}:=N \cap N^{g} \cap \ldots \cap N^{g^{k-1}}$, where $g^{k} \in N . N_{g}$ is $P D^{n}$ by (iv), hence finitely generated. $N$ is countable, therefore so is $\left\{N_{g} ; g \in G\right\}$. As $g \in N_{G}\left(N_{g}\right), G=\bigcup_{g \in G} N_{G}\left(N_{g}\right)$, a countable union. It now suffices to show that, for $P \in \mathscr{P}, N_{G}(P) /\left(N_{G}(P) \cap W\right)$ is countable. $N_{G}(P) / C_{G}(P)$ is isomorphic to a subgroup of Aut $P$, so is countable. So it only remains to prove that $C_{G}(P) /\left(C_{G}(P) \cap W\right)$ is countable. Suppose this is not true. Write $V$ for $C_{G}(P) \cap W$. Plainly $C_{G}(P) / V$ is abelian. For $h \in \zeta(P), k \in \mathbb{N}$, let $P_{k, h}:=\left\{g V \in C_{G}(P) / V: g^{k} V=h V\right\}$. As $\zeta(P)$ is countable, there are only countably many $P_{k, h}$, so by the Dirichlet pigeonhole principle, some $P_{k, h}$ is uncountable. Hence $\exists$ distinct $g_{1} V, g_{2} V \in C_{G}(P) / V$ s.t. $g_{1}^{k} V=g_{2}^{k} V$. Since $C_{G}(P) / V$ is abelian, $\left(g_{1}^{-1} g_{2}\right)^{k} \in V$. By definition of $V, g_{1}^{-1} g_{2} \in V$, hence $g_{1} V=g_{2} V$, so we have a contradiction.

Induction step. We use the notation of (1). Suppose the theorem holds for smaller $r$. Let $H / G_{1}$ be the maximal locally finite subgroup of the union of the centralizers of the $P D$ subgroups of $G_{0} / G_{1}, G_{0} / H \in \overline{\mathscr{X}}_{1}$ by result for $r=1$. We will prove that $H / G_{2}$ is (locally finite)-by-(countable $L O P D^{n}$ )-by-(countable locally finite), so $J \in X_{r-1}$, where $J / G_{2}$ is the given locally finite normal subgroup.

It follows from the inductive hypothesis that $J$ has a characteristic locally finite subgroup $S$ such that $J / S$ is countable.

$$
J=J_{0} \geqq J_{1} \geqq \cdots \geqq J_{r-1}=1
$$

with the $J_{i}$ all normal in $G$ and the $J_{i} / J_{i+1}$ all $L O P D$, since $J \in Y_{r-1}$.

$$
\frac{J}{S}=\frac{J_{0}}{S} \geqq \frac{J_{1} S}{S} \cdots \geqq \frac{J_{r-1} S}{S}=1
$$


is a series in which each $J_{i} S$ is normal in $G$ and each

$$
\frac{J_{i} S}{J_{i+1} S}
$$

is $L O P D^{n}$. If follows that $G / S \in Y_{r}$ as required.

Now it suffices to prove the following.

Lemma 5. $H / G_{2}$ is (locally finite)-by-(countable LOPD $\left.D^{n}\right)-b y$-(countable locally finite).

Proof. Here we may assume that $G_{2}$ is trivial. First, observe that where $\left\{g_{1}, \ldots, g_{k}\right\}$ is a finite subset of $H,\left\langle g_{1}, \ldots, g_{k}\right\rangle \cap G_{1}$ is finitely generated. This follows from the fact that $\left\langle g_{1}, \ldots, g_{k}\right\rangle \cap G_{1}$ is of finite index in $\left\langle g_{1}, \ldots, g_{k}\right\rangle$ which is finitely generated.

Take an arbitrary finite subset $\left\{h_{1}, \ldots, h_{k}\right\}$ of $H$. It follows from the argument above that $\left\langle h_{1}, \ldots, h_{k}\right\rangle \cap G_{1}$ is finitely generated, so lies in an $O P D^{n}$ subgroup $P$ of $G_{1}$. Let $x_{1}, \ldots, x_{l}$ be generators of $P$. Then

$$
P_{2}=\left\langle h_{1}, \ldots, h_{k}, x_{1}, \ldots, x_{l}\right\rangle \cap G_{1}
$$

is finitely generated, so lies inside an $O P D^{n}$ group $P_{1}$ in $G_{1}$. However, it also contains $P$. It follows from [2, Proposition 9.22] that $\left|P_{1}: P\right|$ is finite, therefore $\left|P_{1}: P_{2}\right|$ is finite and $P_{2}$ is an $O P D^{n}$ group. The second isomorphism theorem tells us that

$$
\frac{\left\langle h_{1}, \ldots, h_{k}, x_{1}, \ldots, x_{l}\right\rangle}{P_{2}} \cong \frac{\left\langle h_{1}, \ldots, h_{k}\right\rangle G_{1}}{G_{1}}
$$

therefore $\left\langle h_{1}, \ldots, h_{k}, x_{1}, \ldots, x_{l}\right\rangle$ is a finite extension of $P_{2}$, so is $O P D^{n}$-by-finite. Furthermore, this subgroups is $P D^{n}$, because the definition of the class $\mathrm{X}$ ensures that the order of

$$
\frac{\left\langle h_{1}, \ldots, h_{k}\right\rangle G_{1}}{G_{1}}
$$

is not divisible by char $K$. The proof of Theorem 2 for $r=1$ does not use orientability, hence $H$ has a locally finite normal subgroup $J$ such that $H / J$ is countable locally $\left(O P D^{n}\right.$-by-finite). $H / J$ is the union of an ascending chain of $O P D^{n}$-by-finite groups.

If a $P D^{n}$ group $S$ has a subgroup $T$ of finite index not divisible by char $K$, then the dualizing module $E_{T}$ of $T$ is the restriction to $T$ of the dualising module $E_{S}$ of $S$; considering the corestriction map

$$
H_{n}\left(T, \operatorname{Res}_{T}\left(E_{S}\right)\right) \stackrel{\text { Cor }}{\longrightarrow} H_{n}\left(S, E_{S}\right)
$$

This is onto (see 4.1$)$, therefore $H_{n}\left(T, \operatorname{Res}_{T}\left(E_{S}\right)\right.$ ) is nonzero. The only one-dimensional module $M$ for which $H_{n}(T, M)$ can be nonzero is $E_{T}$. Hence $\operatorname{Res}_{T}\left(E_{S}\right)=E_{T}$. 
It now follows that every $O P D^{n}$-by-finite $P D^{n}$ group has a unique maximal $O P D^{n}$ subgroup. The unique maximal $O P D^{n}$ subgroups of the $O P D^{n}$-by-finite groups in the given ascending chain form an ascending chain; they generate an $L O P D^{n}$ normal subgroup $W / J$ of $H / J$. It is easy to see that $H / W$ is countable locally finite.

Hence $H$ is (locally finite)-by-(countable $L O P D^{n}$ )-by-(countable locally finite), as required.

\subsection{Proof of Theorem 1 for $G$ uncountable}

By Theorem 2, it suffices to prove that if $G$ is locally finite with char $K \notin \mathscr{X}_{G}$ and $M$ is a finite dimensional $K G$-module, then $H^{i}(G, M)=0$ for $i>0$ and

$$
H_{0}(G, M) \stackrel{\cap[\omega]}{\longrightarrow} H_{0}(G, M)
$$

is an isomorphism for $0 \neq[\omega] \in H_{0}(G, K)$.

By a routine calculation, the above cap product is an isomorphism if and only if

$$
M=M^{G} \oplus M g
$$

(Gothic letters represent appropriate augmentation ideals).

Let $N$ be the kernel of the action of $G$ on $M . G / N$ is then a locally finite subgroup of $G L_{s}(K)$ where $s=\operatorname{dim}_{K} M$.

By (9), to show that the given cap product is an isomorphism, it is enough to prove that $M=M^{L} \oplus M$ l.

Choose a maximal linearly independent subset $\left\{g_{1}, \ldots, g_{r}\right\}$ of $L$. This generates a finite group $L_{1}<L . M^{L_{1}} \supseteq M^{L}, M \mathrm{I}_{1} \subseteq M \mathrm{I}$.

Suppose $\exists g \in L$ s.t. $g=\sum_{i=1}^{r} \lambda_{i} g_{i}$ with $\sum_{i=1}^{r} \lambda_{i}=k+1, k \neq 0$. Then for all $m \in M^{L_{1}}$, $m(g-1)=k m$. Thus $M^{L_{1}} \subseteq M$, hence $M=M \mathrm{I}$. $M^{L}=0$ since $m g=(k+1) m \neq m$.

Suppose there is no such $g$. Then for all $g \in G, g=\sum_{i=1}^{r} \lambda_{i} g_{i}$ with $\sum_{i=1}^{r} \lambda_{i}=1$. Therefore $M^{L}=M^{L_{1}}, M I=M I_{1}$. Hence the given cap product is an isomorphism.

$H^{i}(N, M)=0$ for $i=0$ by the Universal Coefficients Theorem. By a spectral sequence corner argument, it now suffices to prove that $H^{i}(G / N, M)=0$ for $i=0$. By a result of Schur (see [12, Corollary 9.4]), $L:=G / N$ is abelian-by-finite. Let $A$ be an abelian normal subgroup of $L$ s.t. $L / A$ is finite. $H^{i}(L, M) \cong H^{i}(A, M)^{L / A}$. $M$ may be expressed as a direct sum of finitely many simple $K A$-modules by [12, Corollary 1.6$]$, hence as their direct product. Therefore $H^{i}(A, M)=\prod_{\lambda \in \Lambda} H^{i}\left(A, M_{\lambda}\right)$ where the $M_{\lambda}$ are simple. Since $A$ acts nontrivially on $M_{\lambda}, H^{i}\left(A, M_{\lambda}\right)=0$ for $i>0$ as required.

\subsection{Homological and cohomological dimensions of groups in $\bar{X}$}

Our construction of $\mathscr{D}_{G}$ was based on that of the module $A$ used in Stammbach's proof [11] that the homological dimension of a group $G$ in the class $C$ is equal to the Hirsch length $h G$, where $C$ is composed of the groups whose factors are locally finite or 
abelian. $C \subseteq \bar{X}$, and for $G \in C, A=\mathscr{D}_{G}$ and $n_{G}=h G$ where $n_{G}:=\sum n(i)$ in the notation of (2). Hence $n_{G}=h_{K}(G)$. This generalizes as follows.

Lemma 7. $n_{G}=\operatorname{hd}_{K}(G)$ for $G \in \overline{\mathscr{X}}$.

Proof. Clearly $n_{G} \leqq$ hd $d_{K}(G)$. For $G$ with $r=1, h_{K}(G)=n_{G}$. Suppose result holds for $r-1$, and consider $G_{1}>\rightarrow G \rightarrow G / G_{1}$. hd $\left(G / G_{1}\right)+\mathrm{hd}_{K}\left(G_{1}\right) \geqq \mathrm{hd}_{K}(G)$ and $n_{G / G_{1}}+n_{G_{1}}=$ $n_{G}$. Since $\operatorname{hd}_{K}\left(G / G_{1}\right)=n_{G / G_{1}}, h_{K}\left(G_{1}\right)=n_{G_{1}}$ by the induction hypothesis, the result follows.

By the method used in the proof of $[4$, Theorem $A]$, it is easily shown that $\operatorname{cd}_{K}\left(G / G_{r-1}\right) \leqq \operatorname{cd}_{K}(G)-n_{G_{r-1}}$. From this, $\operatorname{cd}_{K}(G) \geqq \mathrm{cd}_{K}\left(G / G_{1}\right)+n_{G_{1}}$, by induction on $r-1$.

\subsection{A property of infinitely generated $L O P D^{n}$ groups}

Where $P$ is a $P D^{n}$ subgroup of an infinitely generated $L O P D^{n}$ group $G, P$ has Euler characteristic $\chi(G)=0$.

To show this, assume $G$ is countable. In the notation of (1), $\chi\left(N_{i}\right)=\left|N_{i+1}: N_{i}\right| \chi\left(N_{i+1}\right)$ and $\chi\left(N_{j}\right) \in \mathbb{Z}$.

Suppose $\chi\left(N_{i}\right)$ is nonzero for some $i$. Then it is nonzero for all $i \in \mathbb{N}$, and $\left|\chi\left(N_{0}\right)\right|>\left|N_{i}: N_{0}\right|$ for all $i$, giving a contradiction.

\section{An extension of Theorem 1 for certain groups}

Let $G \in \mathscr{X}$ over $\mathbb{Q}, \mathbb{Q}_{p}$ or any other field $K$ such that every locally finite subgroup of $G L_{n}(K)$ is finite $\left(\mathbb{Q}, \mathbb{Q}_{p}\right.$ have this property; see [12, Theorem 9.33]). Also let $G$ be poly (locally finite without subgroups of finite index, or orientable $P D^{n}$ over $K$ ). Note that soluble minimax groups satisfy these conditions. Then $G$ has restricted Poincare duality on the following full subcategories of $\mathfrak{M}_{K G}$ :

${ }_{c f d} \mathfrak{M}_{G}$ whose objects are the $\stackrel{\lim }{\longrightarrow}\left(M_{j}\right),\left(M_{j}\right)_{j \in I}$ a direct limit system of finite dimensional $K G$-modules;

${ }_{\imath \int d} \mathbb{M}_{G}$ with objects the $\stackrel{\lim }{\longleftarrow}\left(M_{j}\right),\left(M_{j}\right)_{j \in I}$ an inverse limit system of finite dimensional $K G$-modules.

We now prove this. We use the following from [7, Section 2] and [6, Prop. 4]. For $\left(M_{j}\right)_{j \in I}$ a direct limit system of finite dimensional $K G$-modules, the natural map

$$
\stackrel{\lim _{\longrightarrow}}{\longrightarrow} H^{n}\left(G, M_{j}\right) \rightarrow H^{n}\left(G, \stackrel{\lim }{\longrightarrow} M_{j}\right)
$$

is an isomorphism.

For $\left(M_{j}\right)_{j \in I}$ an inverse limit system of finite dimensional $K G$-modules, the natural maps 


$$
\begin{aligned}
& H^{n}\left(G, \stackrel{\lim }{\longleftarrow} M_{j}\right) \rightarrow \stackrel{\lim }{\longleftarrow} H^{n}\left(G, M_{j}\right) \\
& H_{n}\left(G, \stackrel{\lim }{\longleftarrow} M_{j}\right) \rightarrow \stackrel{\lim }{\longleftarrow} H_{n}\left(G, M_{j}\right)
\end{aligned}
$$

are isomorphisms.

We will prove the result for ${ }_{c}{ }_{d} \mathfrak{M}_{G}$; that for ${ }_{i f d} \mathfrak{M}_{G}$ is done by a similar method.

Consider the diagram on the following page, where $[\omega]$ is a nonzero element of

$$
H_{n}\left(G, \mathscr{D}_{G}\right), \phi: M_{i} \rightarrow \stackrel{\lim }{\longrightarrow}\left(M_{j}\right)_{j \in I}
$$

$\eta_{i}$ and $\xi_{i}$ are projections to direct limits, $\gamma_{*}$ is induced from the isomorphism

$$
\gamma: \stackrel{\lim }{\longrightarrow}\left(M_{j} \otimes \mathscr{D}_{G}\right) \rightarrow \stackrel{\lim }{\longrightarrow}\left(M_{j}\right) \otimes \mathscr{D}_{G}
$$

$\alpha, \beta, f$ are the unique maps given by the universal property of direct limit s.t. (1), (2), (3) respectively commute.

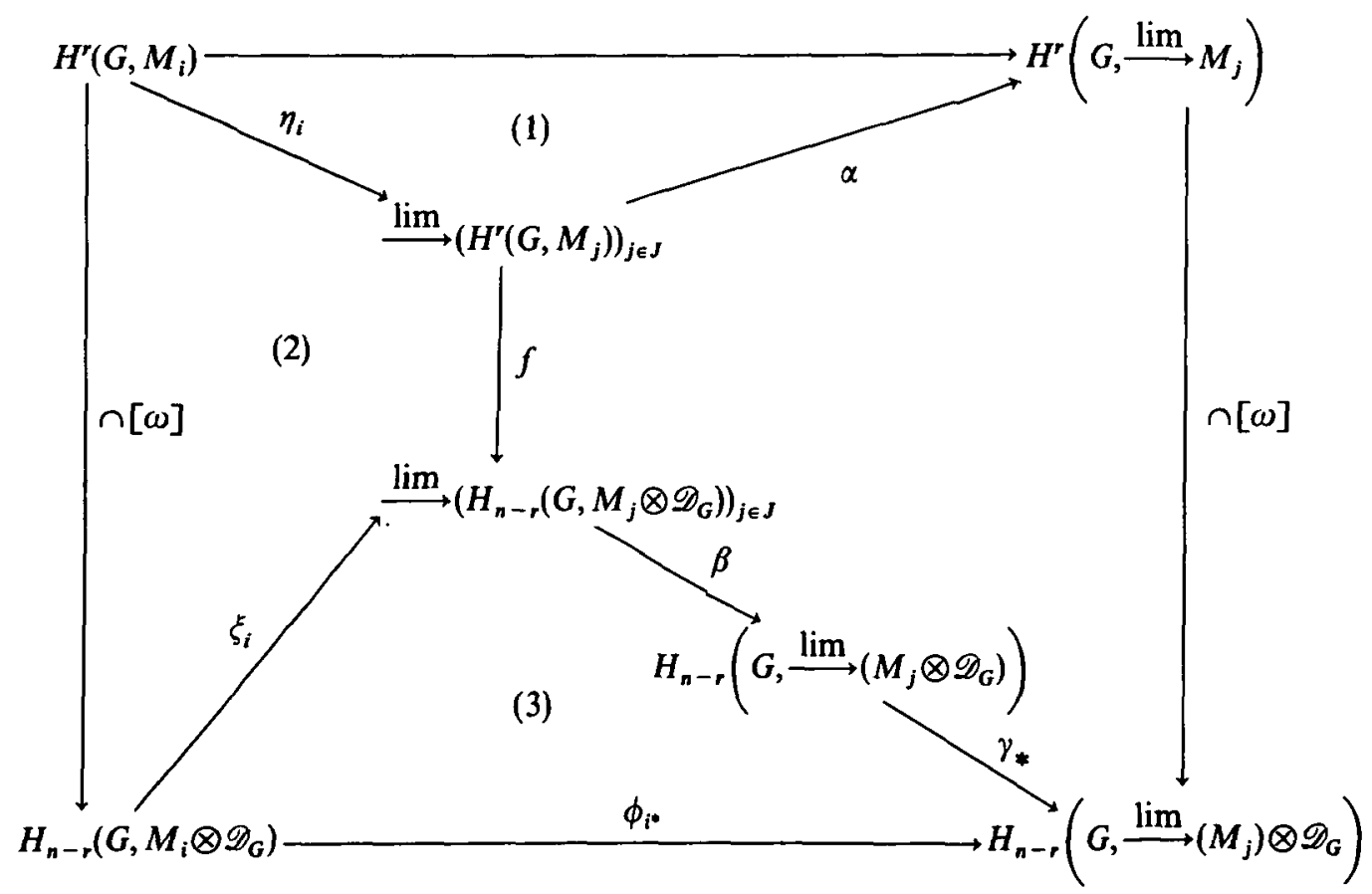


Since (1) commutes, $\cap[\omega] \circ \alpha$ is the unique map given by the universal property such that $\cap[\omega] \circ \alpha \circ \eta_{i}=\cap[\omega] \circ \phi_{i}$, for all $i$. Since (2) and (3) commute, $\gamma * \circ \circ \circ$ is the unique map such that $\gamma * \circ \circ f \circ \eta_{i}=\phi_{i} * \circ[\omega]$ for all $i$. The outer square commutes for all $i \in J$, since $\cap[\omega]$ is natural with respect to coefficient homomorphisms. Hence $\gamma \neq \beta \circ f=\cap[\omega] \circ \alpha$.

$$
H^{r}\left(G, M_{i}\right) \stackrel{\cap[\omega]}{\longrightarrow} H_{n-r}\left(G, M_{i} \otimes \mathscr{D}_{G}\right)
$$

is an isomorphism for all $r, i$ by Theorem 1 , hence $f$ is an isomorphism. $\beta$ is an isomorphism since $H_{s}(G, \ldots)$ commutes with exact colimits; $\alpha$ is an isomorphism by (10). Hence

$$
H^{r}\left(G, \stackrel{\lim _{\longrightarrow}}{\longrightarrow} M_{i} \otimes \mathscr{D}_{G}\right) \stackrel{\cap[\omega]}{\longrightarrow} H_{n-r}\left(G, \stackrel{\lim }{\longrightarrow}\left(M_{i}\right) \otimes \mathscr{D}_{G}\right)
$$

is an isomorphism as required.

\section{Action of $G \in \mathscr{X}$ on its dualizing module over $\mathbb{Q}$}

All known $P D^{n}$ groups over $\mathbb{Q}$ have all $g \in G$ acting on the dualizing module by multiplication by \pm 1 . However, some groups in $\mathscr{X}$ have different actions on $\mathscr{D}_{G}$. For example, for $G=\mathbb{Z}[1 / 2]\}\langle t\rangle$, where $t$ acts on $\mathbb{Z}[1 / 2]$ by multiplication by $2, t$ acts on $\mathscr{D}_{\mathrm{G}}$ by multiplication by 2 .

By the construction of $\mathscr{D}_{G}$ in (2.2.2), it suffices to examine actions of Aut $N$ on $H_{n}(N, \mathbb{Q})$ where $N$ is a $L O P D^{n}$ group.

It is well known that

$$
\left.\begin{array}{l}
H_{n}(N, \mathbb{Q}) \\
\text { over } \mathbb{Q}
\end{array}\right\} \cong\left\{\begin{array}{l}
H_{n}(N, \mathbb{Q}) \\
\text { over } \mathbb{Z}
\end{array}\right.
$$

Over $\mathbb{Z}$, the Universal Coefficients Theorem gives $H_{n}(N, \mathbb{Q}) \cong H_{n}(N, \mathbb{Z}) \otimes_{\mathbb{Z}} \mathbb{Q}$. Now we examine $H_{n}(N, \mathbb{Z})$ for $N$ an $L O P D^{n}$ group. Let $\mathscr{P}$ be the set of all orientable $P D^{n}$ subgroups of $N$. The $H_{n}(P, \mathbb{Z})$ for $P \in \mathscr{P}$ and the corestriction maps between them form a direct limit system, with direct limit $H_{n}(G, \mathbb{Z})$. We will now see what the Cor maps are. For $P \in \mathscr{P}, H_{n}(P, \mathbb{Z}) \cong \mathbb{Z}$.

Lemma 8. Let $P_{i}, P_{j} \in \mathscr{P}$ with $P_{i}<P_{j}$. Let $H_{n}\left(P_{i}, \mathbb{Z}\right)=\langle a\rangle, H_{n}\left(P_{j}, \mathbb{Z}\right)=\langle b\rangle$. Then Cor: $H_{n}\left(P_{i}, Z\right) \rightarrow H_{n}\left(P_{j}, \mathbb{Z}\right)$ is given by

$$
a \rightarrow \pm\left|P_{j}: P_{i}\right| b
$$


Proof. First we will show that the following diagram commutes for all $k \in \mathbb{N}$ and any $K P_{j}$ module $M$, where Sh is the map in Shapiro's Lemma, and $\phi$ is the map

$$
\operatorname{Ind}_{P_{i}}^{P_{j}}(M) \rightarrow M \text { given by }
$$

$$
r \otimes_{K P_{i}} m \rightarrow r m
$$

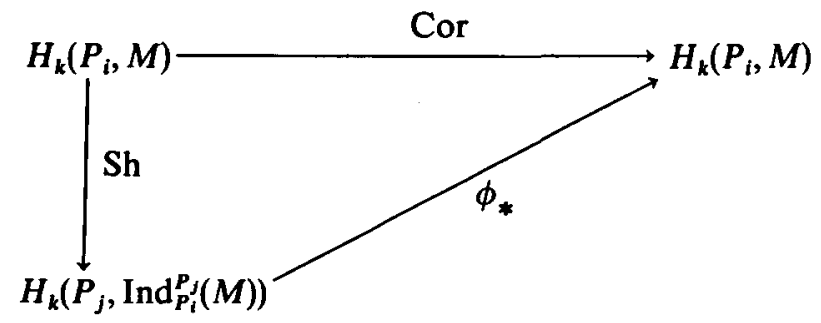

Both $\phi \circ$ Sh and Cor are natural maps from the homology theory $H_{*}\left(P_{i},{ }_{-}\right)$for $P_{j}$ to the universal homology theory $H_{*}\left(P_{j},{ }_{-}\right)$for $P_{j}$, so it suffices to show that (11) commutes for $k=0$. This is easily checked, so (11) commutes.

Now consider

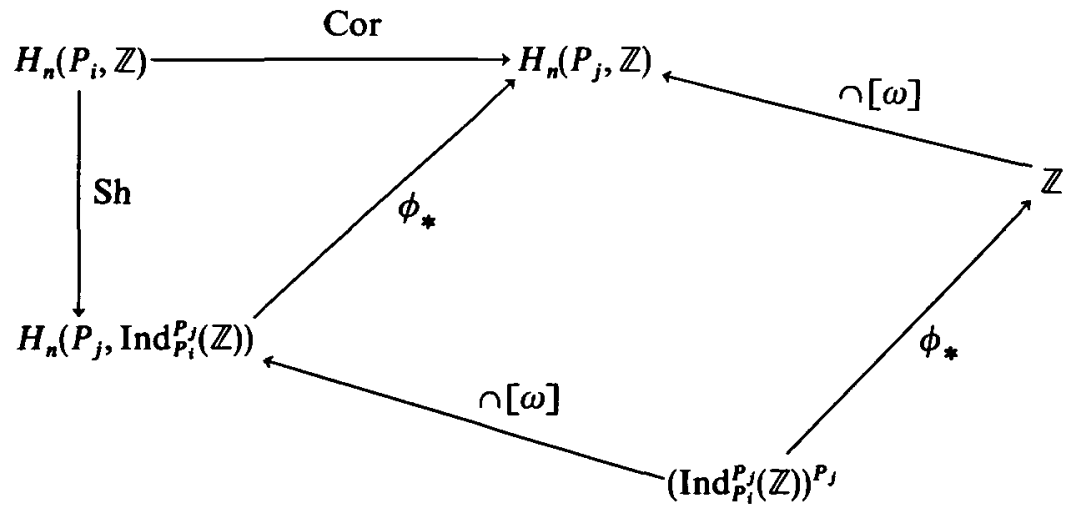

where $n[\omega]$ is the Poincaré duality isomorphism. By naturality of $n$, the square commutes; the triangle commutes by (11).

Take a transversal $1, g_{1}, g_{2}, \ldots, g_{n}$ to $P_{i}$ in $P_{j}$. A general element of $\left(\operatorname{Ind}_{P_{i}}^{P_{j}}(\mathbb{Z})\right)^{P_{j}}$ is of the form $1 \otimes l+g_{1} \otimes l+\cdots+g_{n} \otimes l$ where $l \in \mathbb{Z}$. Hence $\operatorname{Im} \phi_{*}=\left\{\left|P_{j}: P_{i}\right| b\right\}$, and the result follows.

By this lemma, $H_{n}(N, \mathbb{Z}) \cong \mathbb{Z}\left[1 / p_{0}, \ldots, 1 / p_{n}, \ldots\right]$ where $X_{N}=\left\{p_{i}\right\}_{i \in N}$.

$H_{n}(N, \mathbb{Z})$ is preserved setwise by the outer automorphisms of $\mathbb{N}$. Any outer automorphism of $N$ must therefore act on $H_{m}(N, \mathbb{Q})$ by multiplication by $s / t$, where $s, t$ are products of elements of $X_{N}$.

Now we examine $\mathscr{P}$ more closely, to see which actions of this form may occur. We 
may label the $P \in \mathscr{P}$ by elements of $\mathbb{Q}$ as follows. Choose an arbitrary $P \in \mathscr{P}$, and label it as 1 . Label $\boldsymbol{P}_{\boldsymbol{i}} \in \mathscr{P}$ by

$$
\frac{\left|\left\langle P, P_{1}\right\rangle: P\right|}{\left|\left\langle P, P_{1}\right\rangle: P_{1}\right|}
$$

Where $\phi$ is an outer automorphism of $G$, it is easy to see that the action of $\phi$ on $H_{n}(G, \mathbb{Z})$ is multiplication by plus or minus the label of $\phi(P)$; furthermore, this multiplication must preserve the labels.

This tells us that no locally finite group $N$ has an outer automorphism which acts on $\mathscr{D}_{\mathrm{G}}$ by multiplication by $r \neq \pm 1$, for a finite subgroup of lowest order (not necessarily unique) has a label smaller than all the others. By Section 3.1, if $N$ is an uncountable $L O P D^{n}$ group such that $g \in$ Aut $N$ acts by multiplication by $r \neq \pm 1$ on $H_{n}(N, \mathbb{Q})$, there is a countable quotient $N / S$ s.t. $g$ acts by multiplication by $r \neq 1$ on $H_{n}(N / S, \mathbb{Q})$.

We may also deduce that no $P D^{n}$ subgroup $P_{1}$ of an infinitely generated $L O P D^{n}$ group $N$ admitting an outer automorphism which induces multiplication by $r \neq 1$ on $H_{n}(G, \mathbb{Z})$ may be a hyperbolic manifold group. Take $P_{2}:=P_{1} \cap \phi^{-1}\left(P_{1}\right)$, $P_{3}:=\phi\left(P_{2}\right) \subseteq P_{1}$, where $\phi$ is an outer automorphism inducing multiplication by $r \neq \pm 1$ on $H_{n}(G, \mathbb{Z}) . P_{2}$ and $P_{3}$ will be hyperbolic manifold groups with different labels, hence distinct hyperbolic volumes. By rigidity (see [10]), these cannot be isomorphic, so we have a contradiction.

Lemma 9. For $G$ an arbitrary group, let $X$ be a one-dimensional $K G$-module on which $g \in G$ acts by multiplication by $\rho_{g} \in K$. Let $\sigma$ be a field automorphism of $K$. Then $H_{r}(G, X) \cong H_{r}\left(G,{ }_{\sigma} X\right)$ where ${ }_{\sigma} X$ is the one-dimensional $K G$-module on which $g$ acts by multiplication by $\sigma^{-1}\left(\rho_{g}\right)$.

Proof. Let $\alpha:(K G)_{1} \rightarrow(K G)_{2},(K G)_{1}$ and $(K G)_{2}$ both copies of $K G$, be given by $k g \rightarrow \sigma(k) g$. It is well known (see [2, p. 2]) that $\alpha$ induces an isomorphism

$$
\operatorname{Tor}_{n}^{(K G)_{1}}\left({ }^{\alpha} X, K\right) \cong \operatorname{Tor}_{n}^{(K G)_{2}}\left(X,(K G)_{2} \otimes_{(K G)_{1}} K\right)
$$

where ${ }^{\alpha} X$ is $X$ viewed as a $(K G)_{1}$ module via $\alpha$ in the usual way.

We now examine $(K G)_{2} \otimes_{(K G)_{1}} K$ and ${ }^{\star} X$.

The action of $l \in K$ on $(K G)_{2} \otimes_{(K G)_{1}} K$ is given by $l \otimes_{(K G)_{1}} k \rightarrow l \otimes_{(K G)_{1}} k=1 \otimes_{(K G)_{1}} \sigma^{-1}(l) k$. We now write $\sigma(k)_{s}$ for $1 \otimes_{(K G)_{1}} k \cdot k_{s}+l_{s}=(k+l)_{s}$, and the induced $K$-action on $k_{s}$ is $k_{s} \rightarrow(l k)_{s}$. Hence $(K G)_{2} \otimes_{(K G)} K \cong K$.

Take an isomorphism of $K$-modules $\phi: K \rightarrow X$. The action of $g$ on $X$ is given by $g \cdot \phi(k)=\rho_{g} \phi(k)=\phi\left(\rho_{\theta} \cdot k\right)$. $l \in K$ acts on ${ }^{a} X$ by $l^{\cdot a} \phi(k)={ }^{a} \phi(\sigma(l) \cdot k)$, and $g \in G$ acts by $g \cdot{ }^{\alpha} \phi(k)={ }^{\alpha} \phi\left(\rho_{g} \cdot k\right)$. Now write $k_{t}$ for $\alpha_{\phi}(\sigma(k)) . k_{t}+l_{t}=(k+l)_{t}$, the induced action of $l \in K$ is $l \cdot k_{t}=(l k)_{t}$, and the action of $g$ is $k_{t} \rightarrow\left(\sigma^{-1}\left(\rho_{g}\right) \cdot k\right)_{t}$. Hence ${ }^{\alpha} X={ }_{\sigma} X$, and the result follows from (12).

Corollary. Let $G$ be a group with restricted Poincaré duality of dimension $n$ on ${ }_{f d} \mathbb{M}_{\mathrm{RG}}$. 
Then the action of $g \in G$ on the dualizing module $\mathscr{D}_{G}$ is multiplication by an element of the subfield of $K$ which is fixed by all field automorphisms of $K$.

Proof. There is a unique one-dimensional $K G$-module $\mathscr{D}_{G}$ such that $H_{n}\left(G, \mathscr{D}_{G}\right) \cong K$. Hence, for all field automorphisms $\sigma, \sigma^{-} \mathscr{D}_{G} \cong \mathscr{D}_{G}$ as $K G$-modules, hence $\sigma^{-1}\left(\rho_{g}\right)=\rho_{g}$ as required.

\section{REFERENCES}

1. M. F. AтіYah, Characters and cohomology of finite groups, in: Algebraic Topology-a Student's Guide (ed. J. F. Adams) London Math. Soc. Lecture Note Series 4, Cambridge University Press 1972.

2. R. BIERI, Homological Dimension of Discrete Groups (Queen Mary College Maths. Notes, 2nd. edition 1981).

3. K. S. Brown, Cohomology of Groups (Graduate Texts in Maths. 87, Springer, Berlin 1982).

4. D. Gildenhurs and R. Strebel, On the cohomological dimension of soluble groups, Canad. Math. Bull. 24 (1981), 385-392.

5. P. J. Hilton and U. Stammbach, A Course in Homological Algebra (Graduate Texts in Maths. 4, Springer, Berlin 1970).

6. P. H. KRopholler, On finitely generated soluble groups with no large wreath product sections. Proc. London Math. Soc. 49 (1984), 155-169.

7. P. H. Kropholler, The cohomology of soluble groups of finite rank, Proc, London Math. Soc. 53 (1986), 453-473.

8. P. H. Kropholler, Cohomological dimension of soluble groups, J. Pure Appl. Algebra 43 (1986), 281-287.

9. S. Maclane, Homology (Die Grundlehren der Mathematischen Wissenschaften in Einzeldarstellungen, 114, Springer, Berlin 1963). 9-24.

10. J. Milnor, Hyperbolic Geometry: the first 150 years, Bull. Amer. Math. Soc. 6 (1982),

11. U. Stammвасн, On the weak (homological) dimension of the group algebra of solvable groups, J. London Math. Soc. 2 (1970), 567-570.

12. B. A. F. WehrfRITZ, Infinite Linear Groups (Ergebnisse der Mathematik und ihrer Grenzgebiete 76, Springer, Berlin 1973).

School of Mathematical Sciences

Queen Mary and Westfield College

Mile End Road

LONDON E1 4NS 